\title{
Star formation history of early-type galaxies in low density environments ${ }^{\star}$
}

\author{
I. Nuclear line--strength indices \\ M. Longhetti ${ }^{1}$, R. Rampazzo ${ }^{1}$, A. Bressan ${ }^{2}$, and C. Chiosi ${ }^{3}$ \\ 1 Osservatorio Astronomico di Brera, Via Brera 28, I-20121, Milano, Italy \\ 2 Osservatorio Astronomico di Padova, Vic. dell'Osservatorio, 5, I-35122 Padova, Italy \\ 3 Dip. di Astronomia, Univ. di Padova, Vic. dell'Osservatorio, 5, I-35122 Padova, Italy
}

Received July 1; accepted December 8, 1997

\begin{abstract}
This paper is the first of a series (Longhetti et al. 1997a,b) dedicated to the study of the star formation history in early-type galaxies which show fine structures and/or signatures of interaction. It presents nuclear line-strength indices for a sample composed of 21 shell galaxies, from the Malin \& Carter (1983) southern survey, and 30 members of isolated interacting pairs, from the Reduzzi \& Rampazzo (1995) catalogue, located in low density environments.

The spectral range covers $3700 \AA<\lambda<5700 \AA$ at $2.1 \AA$ FWHM resolution. We measure $16 \operatorname{red}(\lambda>4200 \AA)$ indices defined by the Lick Group. Measures have been transformed into the Lick-IDS "standard" system. The procedure has been tested on a set of 5 elliptical galaxies selected from the Gonzalez (1993) sample.

We derive also three blue $(\lambda<4200)$ indices, namely $\Delta(4000 \AA)$ defined by Hamilton $(1985), \mathrm{H}+\mathrm{K}(\mathrm{CaII})$ and $\mathrm{H} \delta / \mathrm{FeI}$ defined by Rose $(1984,1985)$. Blue indices are correlated to the age of the last starburst occurred in a galaxy (Leonardi \& Rose 1996).

The determination of these indices, the estimate of the measurement errors and the correction for the galaxies velocity dispersions are discussed in detail.

In the Appendix A we present the indices for a set of hot stars $(T>10000 \mathrm{~K})$ which may be used for extending W92 fitting functions toward high temperatures.
\end{abstract}

Key words: galaxies: fundamental parameters galaxies: elliptical and lenticular - galaxies: interactions — galaxies: evolution

Send offprint requests to: M. Longhetti

* Based on observations obtained at ESO, La Silla, Chile. Tables 1-8 are also available in electronic form at CDS and Tables 9-15 are only available in electronic form at CDS: via anonymous ftp to cdsarc.u-strasbg.fr (130.79.128.5) or via http://cdsweb.u-strasbg.fr/Abstract.html

\section{Introduction}

N-body simulations of CDM models predict that earlytype galaxies now residing into clusters and groups formed through major merging at redshift $z \approx 3$, while those formed later than $z \approx 1$ have not yet become virialized structures (Governato et al. 1997 and reference therein). Hierarchical clustering scenarios then suggest that earlytype galaxies we see today in clusters and in low density environments have had different histories. Cluster objects are not only ancient but are predicted to have homogeneous properties. Early-type galaxies in low density environments are expected to be younger than the cluster counterparts and more heterogeneous (e.g. most effective galaxy-galaxy interactions take place there). They are then currently interpreted as the final product of processes involving major/minor merging events (see e.g. reviews of Schweizer 1992 and Barnes 1997), but evidences accumulate that both various degrees of dissipation mechanisms (Bender 1997) and weak-interactions (Thomson 1991) have played a significant role in determining the final structure.

Photometric and structural observations of early-type galaxies support hierarchical clustering scenarios showing an environmental dependence. The detection of fine structures and of peculiar kinematics, considered evidence of recent merging/acquisition events, is significantly more frequent among the isolated galaxies then among the cluster members (Malin \& Carter 1983; Schweizer 1992; Reduzzi et al. 1996). The homogeneity of the population of early-type galaxies in clusters is otherwise supported by the Fundamental Plane correlations, examined for several clusters, which may be considered universal to $10 \%-20 \%$ (Djorgovski et al. 1995).

Recently, more efforts, both in the theoretical and in the observational fields, have been dedicated to the determination of sound age indicators for early-type galaxies. 
Line-strength indices have been used to infer the star formation (SF) history of early-type galaxies (see Davies 1995 and references therein), crucial to shed light on the above picture, but firm conclusions have not yet been reached. Buzzoni et al. (1992) suggest that early-type galaxies in the field are almost coeval and characterized by an old stellar population with an average age of $\approx 12$ Gyr (only $2 \%$ of the objects are younger than 5 Gyr). Gonzalez (1993; G93) explains the distribution in the $(\log [\mathrm{MgFe}]-\log \mathrm{H} \beta)$ plane of a sample of 41 "normal" ellipticals (a part of which belongs to clusters e.g. Virgo) as due to quite large age differences (from 4 Gyr to 15 Gyr). Rose (1995) suggests that star formation terminated at an earlier epoch in early-type galaxies in the central regions of dense clusters then in objects in lower density environments. Caveats are raised by Charlot \& Silk (1994) and Bressan et al. (1996) who remark that some line-strength indicators give contradictory evidences about the age of the stellar populations and that the IMF could depend on the galaxy mass. Jorgensen \& Franx (1995) indicate that possible star bursts could be the reason of the age differences among cluster members which contribute significantly to the scatter in the FP. In a recent review on our current understanding of star formation in early-type galaxies, Bender et al. (1996) suggest that the bulk of the stars in the luminous cluster ellipticals must be old and coeval, while the low luminosity early-type galaxies could have smaller ages or extended star formation histories. Bender et al. also suggest that the early-type galaxies in low density environments could be genuinely younger than the luminous cluster ellipticals.

This paper is the first of a series dedicated to the study of a typical population of early-type galaxies in low density environments of galaxies showing present/past signs of interaction. We aim at obtaining information about galaxy formation by investigating their SF history, and the role of the interaction in their evolution. A comparison with the results obtained by other authors in denser environments (Burstein et al. 1984; Pickles 1985; Rose 1985; Rose 1994; Bower et al. 1990; Gonzalez 1993) will further allow us to consider the dependence of the environment on the formation/evolution processes of the early-type galaxies.

The present paper presents the results obtained from the analysis of the spectrophotometric data. It is organized as follows. In Sect. 2 the sample and its characteristics are outlined. Observations and preliminary reduction are presented in Sect. 3. Indices definitions and procedures followed to measure them are given in Sect. 4, where a detailed description of the procedures adopted to transform our measures of the "red" indices in the Lick-IDS "standard" system is also given. Comparisons with stellar and galactic data taken from Worthey (1996, private communication) and from G93 show the final consistency between the two measurements systems. Preliminary conclusions are reported in Sect. 5. In the appendix we present the data relative to a set of hot stars $(T>10000 \mathrm{~K})$ suitable to extend the Worthey (1992; W92) fitting functions.
Further papers will present kinematical information (Longhetti et al. 1997a, Paper II) and ad hoc spectrophotometric models (Longhetti et al. 1997b, Paper III; Longhetti et al. 1998, Paper IV) developed in order to calibrate new spectral indices. These latter will be useful to infer from line-strength indices the presence and the strength of secondary SF events induced by the interaction.

\section{The sample}

At $z \approx 0.5$, nearly half the Hubble time, the bulk of the morphological classes of galaxies visible today in low density environments are already in place (Griffith et al. 1994), but a significant fraction (40\%) of them show peculiarities such as interacting features. Several studies of nearby galaxies (see Schweizer 1992; Reduzzi et al. 1996) conclude that also a large fraction of early-type galaxies shows peculiarities (e.g. fine structures) at the present time. It is useful to remember that while encounters among galaxies in clusters are fast enough to prevent merging (but able to provoke harassment (Moore et al. 1996), interaction in low density environments has a high probability of ending up with a merging event (Barnes 1992). We have selected galaxies showing present/past interaction signatures as typical objects of the low density environment population. Among them, shell galaxies represent a class of objects which show signs of past interaction (minor merging according to Schweizer (1992) or weak-interaction as suggested by Thomson \& Wright (1990) and Thomson (1991), and pair members are objects which are still interacting. We describe below the characteristics of the observed samples. Catalogs from which we have selected them give a description of the environment.

The first sample is composed of 21 almost isolated early-type galaxies characterized by the presence of shells, selected from the Malin \& Carter (1983) catalogue (Table 1). The second one consists of 30 members of isolated binary systems taken from the Reduzzi \& Rampazzo (1995: RR95) catalogue (Table 2) and characterized by various kinds of fine structures. Note that we detect a double nucleus in the galaxy E2400100 (Table 1), unlike other authors that have previously studied this object (Malin \& Carter 1983; Carter et al. 1988). In the following we refer to E2400100 as two separate galaxies, indicated as E2400100 A and B.

Figure 1 shows the distributions of the morphological type and total apparent blue magnitude. A few galaxies in the pair sample are of type later than $T=0$ : their spectra are presented for completeness, both in the present and in the kinematical study (Paper II). Both pairs and shell galaxies are located in low density environments, as mentioned above. In the ESO-LV catalogue (Lauberts \& Valentijn 1989), the $N_{\text {tot }}$ parameter characterizes the surface density of galaxies within one degree from a specific object. Lauberts \& Valentijn (1989) consider that 
Table 1. Shell galaxies

\begin{tabular}{|c|c|c|c|c|c|}
\hline Ident. & $\begin{array}{l}\alpha(1950) \\
\delta(1950)\end{array}$ & $\begin{array}{r}B_{\mathrm{T}} \\
T\end{array}$ & $\begin{array}{l}\mu_{\mathrm{e}}^{\mathrm{B}} \\
A_{\mathrm{e}}^{\mathrm{B}}\end{array}$ & $\begin{array}{l}\text { Comments } \\
\text { Density environment }\end{array}$ & $\begin{array}{c}E(B-V) \\
v_{0}\left[\mathrm{~km} \mathrm{~s}^{-1}\right]\end{array}$ \\
\hline \multirow[t]{2}{*}{ E5390110 } & $00: 20: 24$ & 14.9 & 23.50 & Shells N & 0.0186 \\
\hline & $-19: 18: 39$ & -5.0 & 20.2 & I & \\
\hline \multirow[t]{2}{*}{ E2970340 } & $01: 54: 29$ & 14.5 & 23.70 & Shell E (faint) - W (inner) & 0.0000 \\
\hline & $-39: 29: 19$ & -2.0 & 32.4 & I & \\
\hline E0520160 & 02:00:28 & 13.8 & 22.37 & Shell E-W & 0.0000 \\
\hline NGC 813 & $-68: 41: 19$ & -3.0 & 20.4 & I & 8160 \\
\hline E4800310 & $03: 04: 34$ & 13.7 & 23.14 & Many shells & -0.0116 \\
\hline NGC 1210 & $-25: 54: 38$ & -1.5 & 35.1 & I & 3928 \\
\hline E3570220 & $03: 20: 47$ & 9.1 & 22.80 & Many shells & -0.0046 \\
\hline NGC 1316 & $-37: 23: 06$ & -2.0 & 224.0 & $\mathrm{C}$ (Fornax) & 1739 \\
\hline E1570160 & $04: 14: 39$ & 10.5 & 22.53 & Interacting with NGC1553 & -0.0186 \\
\hline NGC 1549 & $-55: 42: 54$ & -5.0 & 110.0 & G & 1197 \\
\hline E1570170 & 04:15:05 & 10.1 & 21.88 & Interacting with NGC1549 & -0.0209 \\
\hline NGC 1553 & $-55: 54: 12$ & -3.0 & 100.0 & G & 1231 \\
\hline E2500190 & $04: 20: 33$ & 13.2 & 22.26 & & -0.0116 \\
\hline NGC 1571 & $-43: 44: 36$ & -5.0 & 26.9 & I & 4415 \\
\hline E4980010 & $09: 21: 15$ & 12.4 & 21.80 & Chaotic outer structure & 0.0628 \\
\hline NGC 2865 & $-22: 56: 54$ & -5.0 & 32.0 & I & 2611 \\
\hline E5650280 & $09: 35: 23$ & 13.2 & 21.68 & Faint N-S structure & 0.0302 \\
\hline NGC 2945 & $-21: 48: 42$ & -3.0 & 20.0 & I & 4630 \\
\hline E4990160 & $09: 51: 43$ & 12.8 & 23.18 & & 0.0186 \\
\hline NGC 3051 & $-27: 02: 54$ & -2.7 & 49.0 & I & 2552 \\
\hline E5760100 & $13: 10: 20$ & 11.7 & 21.79 & Shells and tail & 0.0512 \\
\hline NGC 5018 & $-19: 15: 18$ & -2.0 & 42.7 & Two companion galaxies & 2794 \\
\hline E1040530 & $19: 20: 38$ & 13.2 & 22.28 & Shell (or loop) NE & 0.0442 \\
\hline NGC 6776 & $-63: 57: 27$ & -3.7 & 28.2 & I & 5450 \\
\hline E3390320 & $20: 02: 53$ & 12.9 & 21.87 & Inner low contrast shells & 0.0600 \\
\hline NGC 6849 & $-40: 20: 31$ & -5.0 & 11.0 & I & 5952 \\
\hline E3410150 & $20: 45: 30$ & 12.3 & 22.36 & Distinct inner structure & 0.0209 \\
\hline NGC 6958 & $-38: 11: 00$ & -3.0 & 43.7 & I & 2627 \\
\hline \multirow[t]{2}{*}{ E1070040 } & 20:59:01 & 13.0 & 22.70 & Shells S and E, faint & 0.0140 \\
\hline & $-67: 23: 00$ & -4.2 & 35.9 & I & 3099 \\
\hline E3420390 & $21: 21: 12$ & 12.4 & 23.41 & Shell NE (wide) & 0.0140 \\
\hline IC 5105 & $-40: 45: 09$ & -3.0 & 66.8 & I & 5382 \\
\hline E4030350 & $21: 46: 47$ & 12.2 & 24.34 & Shell; jet & -0.0116 \\
\hline NGC 7135 & $-35: 06: 37$ & -2.0 & 110.0 & G & 2718 \\
\hline \multirow[t]{2}{*}{ E2890150 } & $22: 15: 07$ & 15.0 & 21.40 & Loop S & -0.0256 \\
\hline & $-42: 56: 23$ & -2.0 & 10.0 & I & \\
\hline \multirow[t]{2}{*}{ E2400100 } & $23: 35: 03$ & 12.5 & 23.23 & Bright gal. extension SE possible shell NW & 0.0000 \\
\hline & $-47: 46: 59$ & -2.0 & 58.2 & $\mathrm{I}$ & 3195 \\
\hline \multirow[t]{2}{*}{ E5380100 } & $23: 55: 06$ & 14.9 & 23.56 & Inner shells; outer dubious & 0.0093 \\
\hline & $-21: 51: 31$ & -3.5 & 23.4 & $\mathrm{I}$ & 10073 \\
\hline
\end{tabular}

$N_{\text {tot }} \geq 9-10$ describes rich environments, e.g. Fornax cluster. With the exclusion of a few objects (namely NGC 1316 in Fornax, NGC 3289, RR 405a and RR 405b) the average $<N_{\text {tot }}>$ value for the pair members is $2 \pm 1.5$, while for shell galaxies is $1.5 \pm 0.9$, indicating that they are in very low density environments.

We consider 5 additional galaxies (Table 3), selected from the G93 sample. We use them as spectrophotometric "templates" to check the transformation of our indices into the Lick-IDS "standard" system (see Sect. 4).
We wish to emphasize that the selected sample complements that of G93. The latter author considers "normal" early-type galaxies, i.e. without fine structures, most of which are in dense environments like the Virgo cluster centre or in groups (Cetus Aries, Libra cloud etc.) (see Tully 1988).

\section{Observations and data reduction}

Long slit spectra have been obtained, during three different runs, at the $1.5 \mathrm{~m}$ ESO telescope (La Silla). 
Table 2. Pair galaxies

\begin{tabular}{|c|c|c|c|c|c|c|c|}
\hline Ident. & $\begin{array}{l}\text { Pair } \\
\text { Ident. }\end{array}$ & $\begin{array}{l}\alpha(1950) \\
\delta(1950)\end{array}$ & $\begin{array}{r}B_{\mathrm{T}} \\
T\end{array}$ & $\begin{array}{l}\mu_{\mathrm{e}}^{\mathrm{B}} . \\
A_{\mathrm{e}}^{\mathrm{B}} .\end{array}$ & Structures & $\begin{array}{l}E(B-V) \\
v_{0}\left[\mathrm{~km} \mathrm{~s}^{-1}\right]\end{array}$ & $\begin{array}{l}\text { Sep. ['] } \\
\Delta V\end{array}$ \\
\hline \multirow[t]{2}{*}{ ESO 2440120} & RR 24a & $01: 15: 55$ & 15.7 & 20.66 & & 0.0000 & 0.3 \\
\hline & & $-44: 43: 37$ & 3.0 & 40.7 & & 6700 & 166 \\
\hline \multirow[t]{2}{*}{ ESO 2440121} & $\mathrm{RR} 24 \mathrm{~b}$ & $01: 15: 56$ & 14.4 & 22.68 & tails and jets & 0.0000 & 0.3 \\
\hline & & $-44: 43: 19$ & -0.3 & 31.6 & & 6866 & 166 \\
\hline \multirow[t]{2}{*}{ ESO 5450400} & RR $62 \mathrm{a}$ & 02:35:53 & 13.8 & 22.51 & inner parts boxy & 0.0093 & 1.7 \\
\hline & & $-20: 22: 55$ & -2.0 & 24.8 & & 1494 & \\
\hline \multirow[t]{2}{*}{ ESO 4860170} & RR101a & 05:01:05 & 14.7 & 21.24 & no interaction sign & 0.0023 & 1.6 \\
\hline & & $-22: 52: 58$ & -2.5 & 10.4 & & 4760 & 130 \\
\hline \multirow[t]{2}{*}{ ESO 4860190} & RR101b & 05:01:10 & 13.6 & 20.51 & no interaction sign & 0.0023 & 1.6 \\
\hline & & $-22: 54: 07$ & -3.5 & 12.6 & & 4630 & 130 \\
\hline \multirow[t]{2}{*}{ ESO 4860290} & RR105a & 05:05:29 & 14.6 & 23.37 & & 0.0070 & 1.6 \\
\hline & & $-23: 07: 55$ & -3.5 & 24.5 & & & \\
\hline \multirow{2}{*}{$\begin{array}{c}\text { ESO } 3750640 \\
\text { NGC3289 }\end{array}$} & RR187b & $10: 31: 50$ & 13.7 & 21.79 & arms & 0.0674 & 2.7 \\
\hline & & $-35: 03: 53$ & -1.0 & 127.4 & & 2702 & 129 \\
\hline \multirow{2}{*}{$\begin{array}{c}\text { ESO } 4400540 \\
\text { NGC4105 }\end{array}$} & RR210a & 12:04:06 & 11.3 & 22.27 & dust & 0.1023 & 1.0 \\
\hline & & $-29: 28: 58$ & -4.0 & 221.3 & & 1820 & 362 \\
\hline \multirow[t]{2}{*}{ ESO 4400560} & RR210b & $12: 04: 10$ & 11.6 & 22.80 & diffuse arms & 0.1023 & 1.0 \\
\hline & NGC4106 & $-29: 29: 23$ & -2.0 & 237.1 & bar, isophote twist & 2182 & 362 \\
\hline \multirow[t]{2}{*}{ ESO 5070450} & $\mathrm{RR} 225 \mathrm{a}$ & $12: 52: 53$ & 12.8 & 22.84 & shells & 0.0767 & 2.2 \\
\hline & & $-26: 33: 10$ & -2.0 & 125.9 & & 4875 & 273 \\
\hline \multirow{2}{*}{ ESO 5070430} & $\mathrm{RR} 225 \mathrm{~b}$ & 12:53:02 & 14.0 & 22.22 & shells? & 0.0767 & 2.2 \\
\hline & & $-26: 32: 16$ & -3.5 & 70.0 & & 4602 & 273 \\
\hline \multirow[t]{2}{*}{ ESO 3860040} & $\mathrm{RR} 278 \mathrm{a}$ & $14: 37: 25$ & 13.7 & 21.55 & shells, dust & 0.0558 & 3.6 \\
\hline & & $-34: 54: 35$ & -2.0 & 71.6 & & 4017 & 441 \\
\hline \multirow[t]{2}{*}{ ESO 2740060} & $\mathrm{RR} 282 \mathrm{~b}$ & $15: 12: 47$ & 14.5 & 22.24 & dust & 0.1419 & 5.3 \\
\hline & & $-43: 49: 47$ & -5.0 & 18.8 & asymmetry & 4458 & 441 \\
\hline \multirow[t]{2}{*}{ ESO 1380290} & $\mathrm{RR} 287 \mathrm{a}$ & $17: 24: 29$ & 12.8 & 23.08 & tails & 0.0744 & 2.9 \\
\hline & & $-62: 24: 18$ & -0.7 & 61.7 & & 4637 & \\
\hline \multirow{2}{*}{$\begin{array}{c}\text { ESO } 1040360 \\
\text { NGC6734 }\end{array}$} & RR297a & 19:02:18 & 13.8 & 22.59 & highly disky & 0.0142 & 2.6 \\
\hline & & $-65: 32: 24$ & -3.0 & 21.6 & & & \\
\hline ESO 1040370 & RR297b & 19:02:35 & 14.3 & 22.72 & & 0.0142 & 2.6 \\
\hline NGC6736 & & $-65: 30: 25$ & -5.0 & 19.5 & & & \\
\hline ESO 1040450 & RR298b & $19: 07: 30$ & 14.7 & 23.32 & & 0.0326 & 0.5 \\
\hline IC 4823 & & $-64: 03: 46$ & -3.0 & 50.7 & & & \\
\hline ESO 2830190 & RR307a & 19:47:47 & 14.8 & 25.60 & arms & 0.0372 & 2.3 \\
\hline & & $-45: 00: 18$ & -1.0 & 65.3 & & & \\
\hline ESO 2830200 & RR307b & $19: 47: 54$ & 14.0 & 23.03 & inner parts boxy & 0.0372 & 2.3 \\
\hline & & $-44: 58: 19$ & -4.0 & 26.0 & outer parts disky & & \\
\hline ESO 4000290 & RR317a & $20: 25: 20$ & 12.7 & 21.60 & warped disk & 0.0186 & 1.1 \\
\hline & & $-36: 11: 34$ & -2.0 & 25.7 & & & \\
\hline ESO 4000300 & RR317b & $20: 25: 22$ & 13.5 & 24.40 & inner part boxy & 0.0186 & 1.1 \\
\hline & & $-37: 12: 36$ & -3.0 & 96.6 & outer parts disky & & \\
\hline ESO 1870230 & RR331a & $20: 48: 12$ & 12.9 & 23.07 & dust & 0.0558 & 10.9 \\
\hline & & $-57: 15: 28$ & -0.4 & 50.1 & asymmetry & 3402 & 25 \\
\hline ESO 5330310 & RR381a & $22: 25: 49$ & 13.0 & 22.96 & tails and dust & -0.0023 & 0.5 \\
\hline & & $-25: 06: 00$ & -2.0 & 46.8 & & 4706 & 359 \\
\hline ESO 1090220 & RR387a & $22: 44: 00$ & 12.1 & 22.27 & & 0.0023 & 0.5 \\
\hline IC 5250 & & $-65: 19: 19$ & -2.0 & 166.0 & & 2947 & \\
\hline ESO 1090221 & RR387b & $22: 44: 04$ & 12.2 & 22.74 & & 0.0023 & 0.5 \\
\hline & & $-65: 19: 11$ & -3.0 & 166.0 & & & \\
\hline ESO 2910280 & RR397b & $23: 30: 35$ & 12.2 & 22.51 & & 0.000 & 0.8 \\
\hline IC 5328 & & $-45: 17: 34$ & -2.5 & 175.8 & & 3136 & 977 \\
\hline ESO 4710190 & $\mathrm{RR} 405 \mathrm{a}$ & $23: 45: 09$ & 13.4 & 24.14 & & 0.0093 & 0.4 \\
\hline & & $-28: 25: 01$ & -5.0 & 197.2 & & 8631 & 24 \\
\hline ESO 4710191 & RR405b & $23: 45: 10$ & 13.5 & 23.24 & & 0.0093 & 0.4 \\
\hline & & $-28: 24: 50$ & -4.0 & 147.9 & & 8607 & 24 \\
\hline ESO 4710470 & RR409a & $23: 53: 49$ & 15.2 & 22.58 & & 0.0000 & 0.3 \\
\hline IC 5364 & & $-29: 18: 07$ & 0.3 & 57.5 & & & \\
\hline ESO 4710471 & RR409b & $23: 53: 51$ & 14.7 & 22.81 & boxy, isophote twist & 0.0000 & 0.3 \\
\hline & & $-29: 18: 03$ & -3.0 & 56.2 & & 9186 & \\
\hline
\end{tabular}



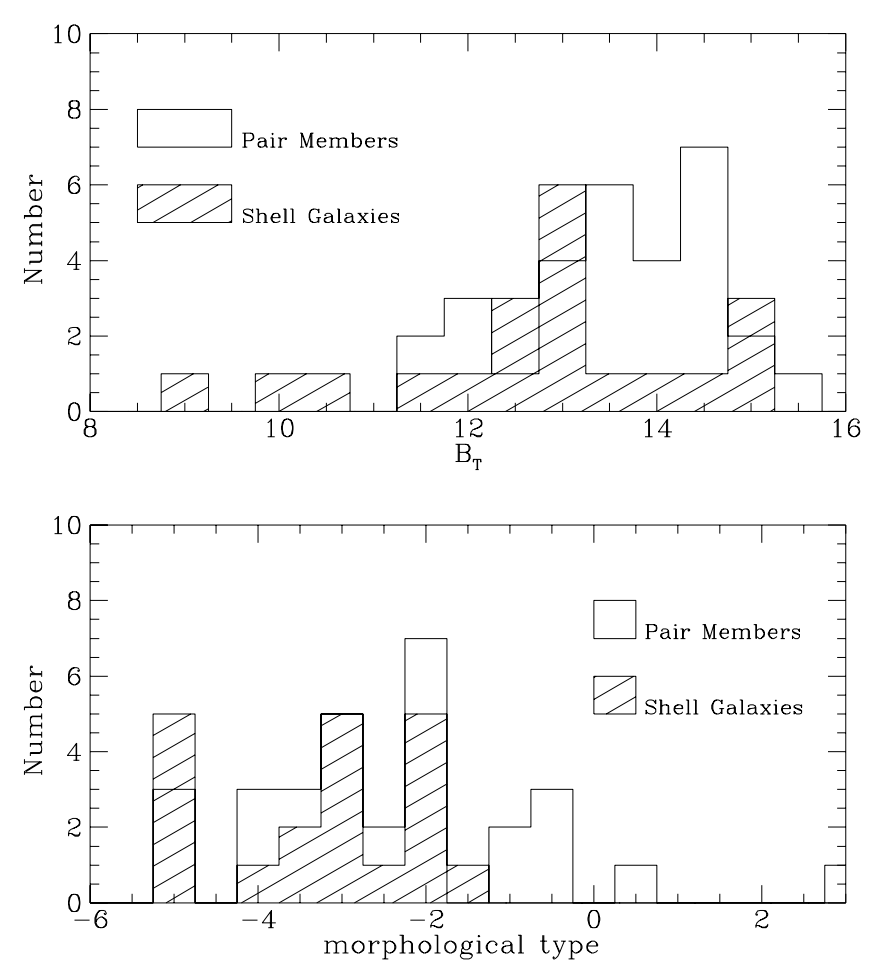

Fig. 1. Distribution of magnitudes (top panel) and of morphological types (bottom panel) within the two subsamples

Table 3. "Template galaxies"

\begin{tabular}{crrc}
\hline Ident. & $\alpha(2000)$ & $B_{\mathrm{T}}$ & $E(B-V)$ \\
& $\delta(2000)$ & $T$ & $v_{0}\left[\mathrm{~km} \mathrm{~s}^{-1}\right]$ \\
\hline \multirow{2}{*}{ NGC 584 } & $01: 31: 21$ & 11.4 & 0.030 \\
& $-06: 52: 06$ & -5.0 & 1864 \\
NGC7562 & $23: 15: 57$ & 12.6 & 0.050 \\
& $+06: 41: 15$ & -5.0 & 3636 \\
NGC7619 & $23: 20: 15$ & 12.1 & 0.040 \\
& $+08: 12: 23$ & -5.0 & 3804 \\
NGC7626 & $23: 20: 42$ & 12.2 & 0.040 \\
& $+08: 13: 02$ & -5.0 & 3423 \\
NGC7785 & $23: 55: 19$ & 12.6 & 0.040 \\
& $+05: 54: 43$ & -5.0 & 3849 \\
\hline
\end{tabular}

The telescope was equipped with a Boller \& Chivens spectrograph and a UV coated CCD Fa2048L $(2048 \times 2048)$ camera. Parameters of the observations and range of the variations of the seeing for each run are reported in Table 4 . Table 5 details: the object identification (1), the observing run (2), the slit position angle (oriented NE) (3), the exposure time (4), the spectrum quality (5) and the galaxy portion enclosed in $5^{\prime \prime}$ in terms of equivalent diameter $D_{\mathrm{e}}^{\mathrm{B}}$ (i.e. the diameter enclosing half of the total light) (6). The quality of the spectrum is defined as the average $S / N\left(\AA^{-1}\right)$ ratio in the wavelength range $4000 \AA-5000 \AA$. Higher $S / N$ spectra are indicated with $3(S / N>20)$ while lower ones are indicated with $1(S / N \leq 10)$. Galaxies observations were split into multiple exposures (typically three) in order to perform cosmic rays cleaning by a me- dian average among the frames. The slit has been oriented along the major axis for almost all the objects. Some pairs have been observed along the line connecting the nuclei. $\mathrm{He}-\mathrm{Ar}$ lamps were taken before and after each exposure to allow an accurate wavelength calibration.

Each frame has been treated separately. Pre-reduction, wavelength calibration and sky subtraction have been performed using the IRAF package. Relative flux calibration has been obtained with the use of a sequence of standard stars. Comparing calibrated stellar spectra common to different nights and different runs, we estimate that the flux uncertainty is about $1.0 \%$ for data relative to the run $n .1$, about $0.4 \%$ for those relative to the run $n .2$ and $3.5 \%$ for those relative to the last run.

Before being calibrated in flux, frames have been corrected for the atmospheric extinction (using the ESO La Silla coefficients) and for the Galactic extinction (using the values of $E_{(B-V)}$ from Burstein \& Heiles 1982). Multiple spectra, relative to the same object, have then been coadded: their alignment was better than 0.5 pixel. From each flux calibrated spectrum, the portion corresponding to the central $5^{\prime \prime}$ of the galaxy nucleus has been extracted and co-added producing a one dimensional spectrum. Objects spectra have been convolved with a gaussian curve $(\sigma=3.37 \AA)$ in order to degrade their resolution up $8.2 \AA$ (FWHM), to match the Lick-IDS data resolution (see Sect. 4).

\section{Nuclear line-strength indices}

The spectrum of a galaxy is produced by the sum of the spectral characteristics of its stellar content. The relative contribution given by each stellar type strongly depends on the wavelength considered. Buzzoni $(1989,1995)$ and Worthey $(1992,1994)$ have given estimates about the sensitivity of several indices to the metallicity or to the age of a stellar population. From these studies it is possible to infer that most of the line-strength indices in the LickIDS System are sensible to the galaxy metallicity and that only the $\mathrm{H} \beta$ line-strength index is connected in a significant way to the galaxy age. Since we are investigating the effect of interaction on the stellar components of our sample galaxies, we decided to measure and calibrate a set of line-strength indices sensible to recent star Formation (SF), to which we refer as "blue" indices, in addition to those in the standard Lick-IDS system ("red indices"). The details of the line-strength indices are described in the sections below.

\subsection{Red and blue indices}

We indicate as red 16 line-strength indices defined in the wavelength range between $4200 \AA$ and $5500 \AA$. Atomic, $I_{\mathrm{a}}$, and molecular, $I_{\mathrm{m}}$, indices are defined by the following 
Table 4. Observing parameters

\begin{tabular}{|c|c|c|c|}
\hline & Run 1 & Run 2 & Run 3 \\
\hline Date of observations & 7-9 March & 1-5 August & 14-21 August \\
\hline Observer & Longhetti, M. & Bonfanti, P. & Longhetti, M. \\
\hline Spectrograph & $\mathrm{B} \& \mathrm{C}$ & $\mathrm{B} \& \mathrm{C}$ & $\mathrm{B} \& \mathrm{C}$ \\
\hline Detector & Fa $2048 \mathrm{~L}$ & Fa $2048 \mathrm{~L}$ & Fa $2048 \mathrm{~L}$ \\
\hline Pixel size $\left(\mu \mathrm{m}^{2}\right)$ & 15 & 15 & 15 \\
\hline Scale along the slit $\left({ }^{\prime \prime} \mathrm{px}^{-1}\right)$ & 0.61 & 0.61 & 0.61 \\
\hline Slit length $\left({ }^{\prime}\right)$ & 4.2 & 4.2 & 4.2 \\
\hline Slit width $\left({ }^{\prime \prime}\right)$ & 2.0 & 2.0 & 2.0 \\
\hline Dispersion $\left(\AA^{\circ} m^{-1}\right)$ & 66 & 66 & 66 \\
\hline Spectral resolution (FWHM at $5200 \AA)(\AA)$ & 2.1 & 2.1 & 2.1 \\
\hline Spectral range $[\AA]$ & $3750-5750$ & $3704-5710$ & $3728-5747$ \\
\hline Instrumental velocity dispersion $\sigma\left(\mathrm{km} \mathrm{s}^{-1}\right)$ & 51 & 51 & 51 \\
\hline Standard stars spectral type & K0-K2 III & K0-K2 III & K0-K2 III \\
\hline Seeing (FWHM) [arcsec] & $1.0-1.5$ & $1.0-1.5$ & $<2$ \\
\hline
\end{tabular}

Table 5. Journal of galaxies observations

\begin{tabular}{|c|c|c|c|c|c|c|c|c|c|c|c|}
\hline Ident. & Run & $\begin{array}{l}\text { Slit } \\
\text { PA }\end{array}$ & $t_{\exp }$ & $\begin{array}{l}S / N \\
\text { class }\end{array}$ & $5\left(^{\prime \prime}\right) / D_{\mathrm{e}}^{\mathrm{B}}$ & Ident. & Run & $\begin{array}{c}\text { Slit } \\
\text { PA }\end{array}$ & $t_{\text {exp }}$ & $\begin{array}{l}S / N \\
\text { class }\end{array}$ & $5\left(^{\prime \prime}\right) / D_{\mathrm{e}}^{\mathrm{B}}$ \\
\hline E 5390100 & $2-3$ & 6 & 280 & 1 & 0.20 & E 2400100 & 3 & 133 & 130 & 2 & \\
\hline E 2970340 & 3 & 88 & 210 & 1 & 0.12 & E 5380100 & 3 & 168 & 180 & 2 & 0.21 \\
\hline NGC 584 & 2 & 60 & 100 & 3 & 0.08 & $\mathrm{RR} 24 \mathrm{a} / \mathrm{b}$ & 2 & 1 & 120 & $1 / 1$ & $0.30 / 0.17$ \\
\hline NGC 813 & 3 & 103 & 135 & 3 & 0.20 & RR 62a & 2 & 37 & 120 & 2 & 0.17 \\
\hline NGC 1210 & 3 & 121 & 135 & 2 & 0.13 & RR 101a/b & 2 & 161 & 95 & $1 / 3$ & $0.33 / 0.17$ \\
\hline NGC 1316 & 3 & 45 & 80 & 3 & 0.02 & $\mathrm{RR} 105 \mathrm{a}$ & 2 & 140 & 95 & 1 & 0.17 \\
\hline NGC 1549 & 3 & 158 & 90 & 3 & 0.04 & $\mathrm{RR} 187 \mathrm{~b}$ & 1 & 153 & 135 & 2 & 0.10 \\
\hline NGC 1553 & 3 & 155 & 90 & 3 & 0.04 & RR 210a & 1 & 118 & 70 & 2 & 0.05 \\
\hline NGC 1571 & 3 & 177 & 150 & 3 & 0.15 & $\mathrm{RR} 210 \mathrm{~b}$ & 1 & 55 & 75 & 2 & 0.04 \\
\hline NGC 2865 & 1 & 146 & 100 & 3 & 0.14 & $\mathrm{RR} 225 \mathrm{a}$ & 1 & 170 & 105 & 2 & 0.11 \\
\hline NGC 2945 & 1 & 157 & 115 & 2 & 0.23 & $\mathrm{RR} 225 \mathrm{~b}$ & 1 & 45 & 105 & 1 & 0.25 \\
\hline NGC 3051 & 1 & 33 & 105 & 2 & 0.10 & $\mathrm{RR} 278 \mathrm{a}$ & 1 & 23 & 105 & 1 & 0.24 \\
\hline NGC 5018 & 1 & 100 & 90 & 3 & 0.11 & $\mathrm{RR} 282 \mathrm{~b}$ & 2 & 170 & 120 & 2 & 0.22 \\
\hline NGC 6776 & 3 & 12 & 140 & 3 & 0.16 & RR 287a & 2 & 28 & 105 & 1 & 0.06 \\
\hline NGC 6849 & 3 & 25 & 150 & 2 & 0.06 & RR 297a/b & 2 & 37 & 105 & $1 / 1$ & $0.21 / 0.22$ \\
\hline NGC 6958 & 3 & 87 & 130 & 3 & 0.10 & RR 298b & 2 & 11 & 120 & 1 & 0.16 \\
\hline E 1070040 & 3 & 139 & 145 & 3 & 0.13 & $\mathrm{RR} 307 \mathrm{a} / \mathrm{b}$ & 2 & 28 & 105 & $1 / 2$ & $0.06 / 0.15$ \\
\hline IC 5105 & 2 & 40 & 115 & 2 & 0.06 & RR 317a/b & 3 & 165 & 160 & $3 / 1$ & $0.13 / 0.05$ \\
\hline NGC 7135 & 3 & 33 & 135 & 3 & 0.04 & RR 331a & 2 & 116 & 105 & 1 & 0.08 \\
\hline NGC 7562 & 2 & 83 & 100 & 2 & 0.10 & RR 381a & 2 & 69 & 115 & 2 & $0.10 / 0.06$ \\
\hline NGC 7619 & 2 & 30 & 100 & 2 & 0.08 & $\mathrm{RR} 387 \mathrm{a} / \mathrm{b}$ & 3 & 79 & 140 & $3 / 3$ & $0.08 / 0.07$ \\
\hline NGC 7626 & 2 & 0 & 105 & 2 & 0.07 & RR 397b & 3 & 42 & 105 & 3 & 0.08 \\
\hline NGC 7785 & 2 & 143 & 105 & 2 & 0.09 & $\mathrm{RR} 405 \mathrm{a} / \mathrm{b}$ & 3 & 63 & 140 & $2 / 2$ & $0.04 / 0.06$ \\
\hline E 2890150 & 3 & 132 & 282 & 2 & 0.48 & $\mathrm{RR} 409 \mathrm{a} / \mathrm{b}$ & 2 & 85 & 130 & $1 / 1$ & $0.23 / 0.23$ \\
\hline
\end{tabular}


Table 6. Definition of the Lick-IDS indices

\begin{tabular}{|c|c|c|c|c|}
\hline Index & $\begin{array}{l}\text { Spectral } \\
\text { Features }\end{array}$ & $\lambda_{\mathrm{I}_{1}}-\lambda_{\mathrm{I}_{2}}[\AA]$ & $\begin{array}{l}\lambda_{\mathrm{b}_{1}}-\lambda_{\mathrm{b}_{2}}[\AA] \\
\lambda_{\mathrm{r}_{1}}-\lambda_{\mathrm{r}_{2}}[\AA]\end{array}$ & $\begin{array}{l}\text { Type } \\
\text { [unit] }\end{array}$ \\
\hline $\mathrm{CN}$ & CN, TiI, FeI & $4143.375-4178.375$ & $\begin{array}{l}4081.375-4118.875 \\
4245.375-4285.375\end{array}$ & $\begin{array}{r}\text { Molecular } \\
\text { [Mag] }\end{array}$ \\
\hline $\mathrm{CN} 2$ & CN, TiI, FeI & $4143.375-4178.375$ & $\begin{array}{l}4085.125-4097.625 \\
4245.375-4285.375\end{array}$ & $\begin{array}{r}\text { Molecular } \\
\text { [Mag] }\end{array}$ \\
\hline $\mathrm{Ca} 4227$ & $\mathrm{CaI}, \mathrm{FeI}$ & $4223.500-4236.00$ & $\begin{array}{l}4212.250-4221.000 \\
4242.250-4252.250\end{array}$ & $\begin{array}{r}\text { Atomic } \\
{[\AA]}\end{array}$ \\
\hline G4300 & CHband, FeI & $4282.625-4317.625$ & $\begin{array}{l}4267.625-4283.875 \\
4320.125-4336.375\end{array}$ & $\begin{array}{r}\text { Atomic } \\
{[\AA]}\end{array}$ \\
\hline $\mathrm{Fe} 4383$ & $\mathrm{FeI}$ & $4370.375-4421.625$ & $\begin{array}{l}4360.375-4371.625 \\
4444.125-4456.625\end{array}$ & $\begin{array}{r}\text { Atomic } \\
{[\AA]}\end{array}$ \\
\hline $\mathrm{Ca} 4455$ & $\begin{array}{l}\text { CaI, FeI } \\
\text { NiI, TiII }\end{array}$ & $4453.375-4475.875$ & $\begin{array}{l}4447.125-4455.875 \\
4478.375-4493.375\end{array}$ & $\begin{array}{r}\text { Atomic } \\
{[\AA]}\end{array}$ \\
\hline Fe4531 & FeI, TiI & $4515.500-4560.500$ & $\begin{array}{l}4505.500-4515.500 \\
4561.750-4580.500\end{array}$ & $\begin{array}{r}\text { Atomic } \\
{[\AA]}\end{array}$ \\
\hline Fe4668 & FeI, CrI, TiI & $4635.250-4721.500$ & $\begin{array}{l}4612.750-4631.500 \\
4744.000-4757.750\end{array}$ & $\begin{array}{r}\text { Atomic } \\
{[\AA]}\end{array}$ \\
\hline $\mathrm{H} \beta$ & $\mathrm{H} \beta$ & $4847.875-4876.625$ & $\begin{array}{l}4827.875-4847.875 \\
4876.625-4891.625\end{array}$ & $\begin{array}{r}\text { Atomic } \\
{[\AA]}\end{array}$ \\
\hline Fe5015 & FeI, TiI, NiI & $4977.750-5054.000$ & $\begin{array}{l}4946.500-4977.750 \\
5054.000-5065.250\end{array}$ & $\begin{array}{r}\text { Atomic } \\
{[\AA]}\end{array}$ \\
\hline$M \mathrm{~g}_{1}$ & $\mathrm{MgH}, \mathrm{FeI}$ & $5069.125-5134.125$ & $\begin{array}{l}4895.125-4957.625 \\
5301.125-5366.125\end{array}$ & $\begin{array}{r}\text { Molecular } \\
\text { [Mag] }\end{array}$ \\
\hline$M \mathrm{~g}_{2}$ & $\mathrm{MgH}, \mathrm{MgI}$ & $5154.125-5196.625$ & $\begin{array}{l}4895.125-4957.625 \\
5301.125-5366.125\end{array}$ & $\begin{array}{r}\text { Molecular } \\
\text { [Mag] }\end{array}$ \\
\hline$M \mathrm{~g}_{\mathrm{b}}$ & $\mathrm{MgI}$ & $5160.125-5192.625$ & $\begin{array}{l}5142.625-5161.375 \\
5191.375-5206.375\end{array}$ & $\begin{array}{r}\text { Atomic } \\
{[\AA]}\end{array}$ \\
\hline $\mathrm{Fe} 5270$ & FeI, CaI & $5245.650-5285.650$ & $\begin{array}{l}5233.150-5248.150 \\
5285.650-5318.150\end{array}$ & $\begin{array}{r}\text { Atomic } \\
{[\AA]}\end{array}$ \\
\hline Fe5335 & $\begin{array}{r}\text { FeI, CrI } \\
\text { CaI, TiII }\end{array}$ & $5312.125-5352.125$ & $\begin{array}{l}5304.625-5315.875 \\
5353.375-5363.375\end{array}$ & $\begin{array}{r}\text { Atomic } \\
{[\AA]}\end{array}$ \\
\hline Fe5406 & FeI, CrI & $5387.500-5415.000$ & $\begin{array}{l}5376.250-5387.500 \\
5415.000-5425.000\end{array}$ & $\begin{array}{r}\text { Atomic } \\
{[\AA]}\end{array}$ \\
\hline
\end{tabular}

formulas (G93):

$I_{\mathrm{a}}=\int_{\lambda_{\mathrm{I}_{1}}}^{\lambda_{\mathrm{I}_{2}}}\left(1-\frac{F(\lambda)}{C(\lambda)}\right) \mathrm{d} \lambda$

and

$I_{\mathrm{m}}=-2.5 \log _{10} \frac{\int_{\lambda_{\mathrm{I}_{1}}}^{\lambda_{\mathrm{I}_{2}}} \frac{F(\lambda)}{C(\lambda)} \mathrm{d} \lambda}{\left(\lambda_{\mathrm{I}_{2}}-\lambda_{\mathrm{I}_{1}}\right)}$.

The $C(\lambda)$ function represents the spectral continuum obtained by interpolating fluxes in two windows chosen near the two sides of the feature:

$C(\lambda)=F_{\mathrm{b}} \frac{\lambda_{\mathrm{r}}-\lambda}{\lambda_{\mathrm{r}}-\lambda_{\mathrm{b}}}+F_{\mathrm{r}} \frac{\lambda-\lambda_{\mathrm{b}}}{\lambda_{\mathrm{r}}-\lambda_{\mathrm{b}}}$

$$
\begin{aligned}
& F_{\mathrm{b}}=\frac{\int_{\lambda_{\mathrm{b}_{1}}}^{\lambda \mathrm{b}_{2}} F(\lambda) \mathrm{d} \lambda}{\left(\lambda_{\mathrm{b}_{2}}-\lambda_{\mathrm{b}_{1}}\right)} \\
& F_{\mathrm{r}}=\frac{\int_{\lambda_{\mathrm{r}_{1}}}^{\lambda \mathrm{r}_{2}} F(\lambda) \mathrm{d} \lambda}{\left(\lambda_{\mathrm{r}_{2}}-\lambda_{\mathrm{r}_{1}}\right)}
\end{aligned}
$$
$\lambda_{\mathrm{r}}=\frac{\left(\lambda_{\mathrm{r} 1}+\lambda_{\mathrm{r} 2}\right)}{2}$
$\lambda_{\mathrm{b}}=\frac{\left(\lambda_{\mathrm{b} 1}+\lambda_{\mathrm{b} 2}\right)}{2}$.

The adopted spectral bandpasses of the red indices are taken from Burstein et al. (1984) and G93 and are detailed in Table 6 . Spectral ranges involved by the indices measurements are shown in the Figs. 2a,b (shaded areas) superimposed on a set of stellar spectra. Different spectral types are selected in order to emphasize the variations of 


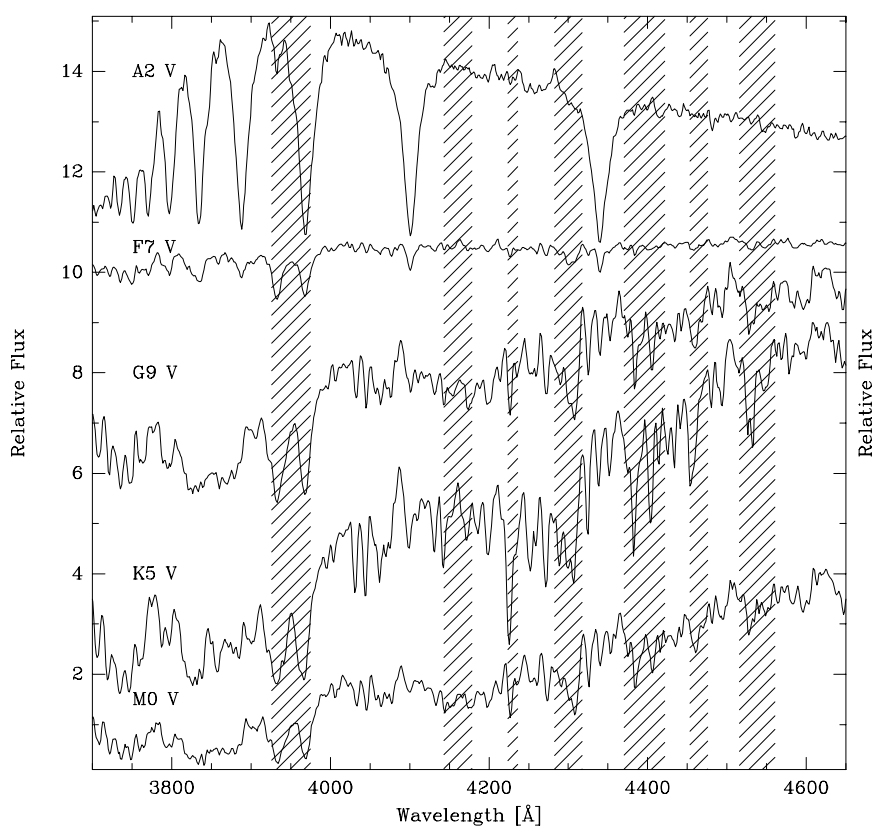

a)

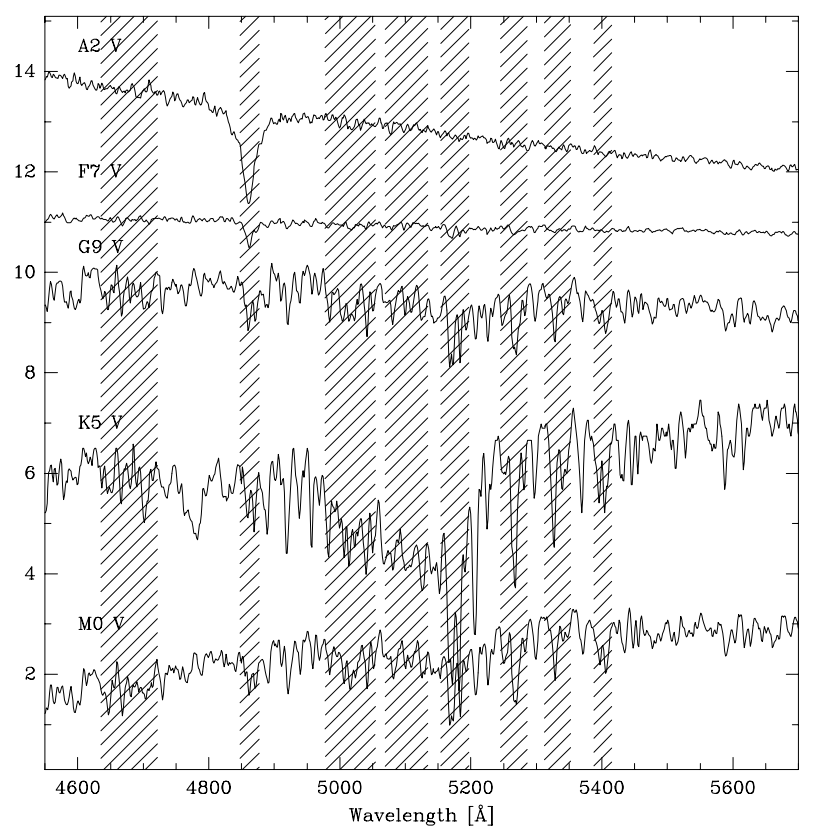

b)

Fig. 2. Wavelength range (shaded area) of line-strength spectral indices superimposed onto different stellar spectra. The shaded areas are intended to highlight the variations of the spectral features as a function of spectral types
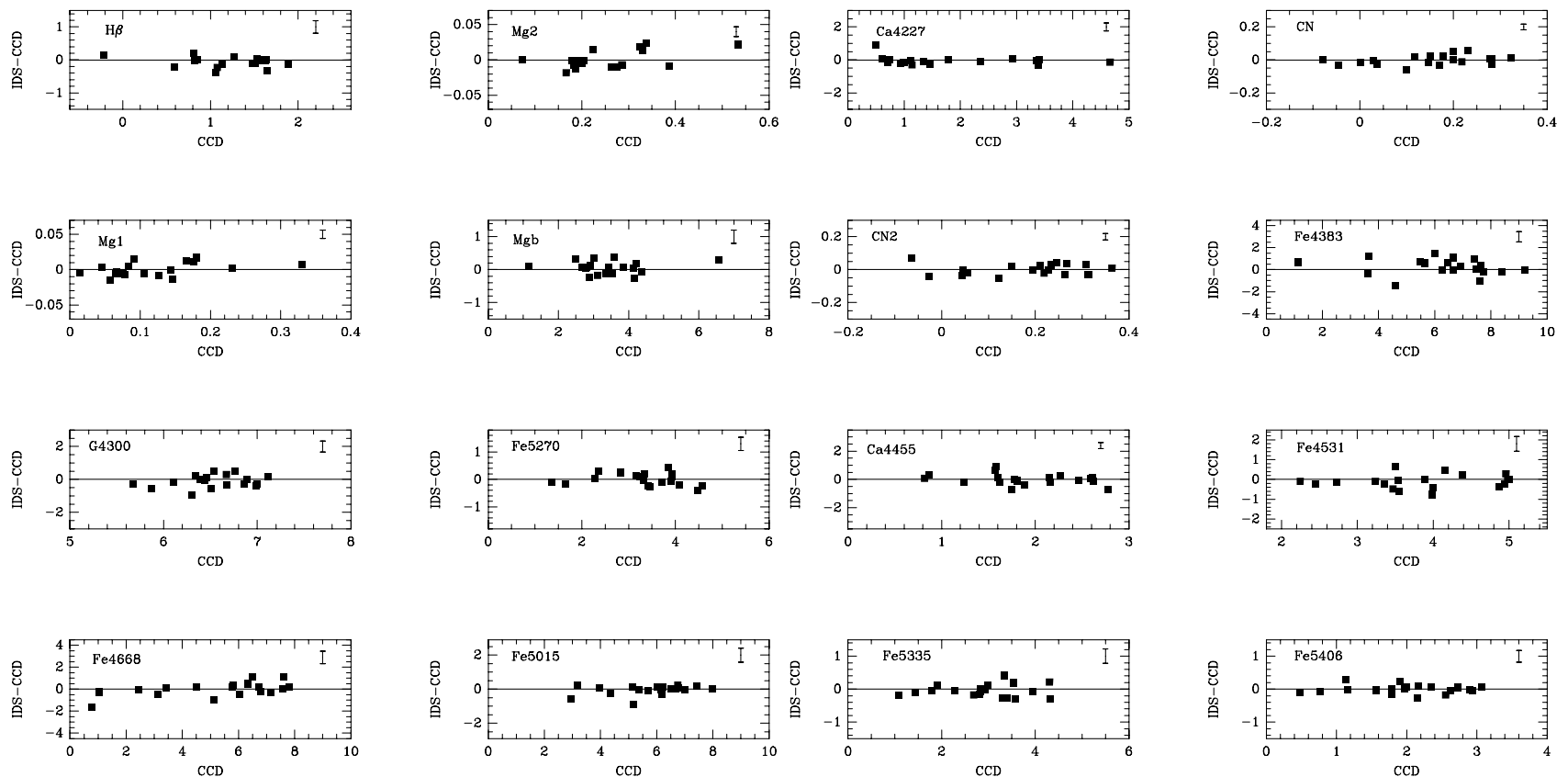

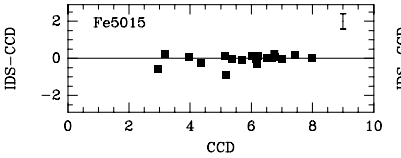

a)

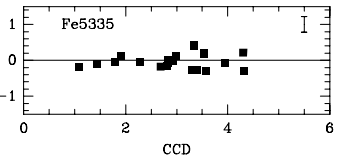

b)

Fig. 3. Comparison between our CCD measure (after the complete transformation to the Lick system) and W92 IDS data on the common sample of 19 stars. In each diagram, the error bar refers to the average value obtained on the whole sample. The dimension of the error bar along the abscissa is of the same order as the symbols 
Table 7. Definition of the "blue" indices

\begin{tabular}{rrcc}
\hline Index & $\begin{array}{r}\text { Spectral } \\
\text { Features }\end{array}$ & $\lambda_{1}-\lambda_{2}[\AA]$ & $\begin{array}{l}\lambda_{3}-\lambda_{4}[\AA] \\
\lambda_{5}-\lambda_{6}[\AA]\end{array}$ \\
\hline $\mathrm{H}+\mathrm{K}(\mathrm{CaII})$ & $\mathrm{H}$ e K $(\mathrm{CaII})$ & $3926.0-3940.0$ & $3961.0-3975.0$ \\
$\mathrm{H} \varepsilon$ & & \\
$\mathrm{H} \delta / \mathrm{FeI}$ & $\mathrm{H} \delta, \mathrm{FeI}$ & $4095.0-4108.0$ & $4041.0-4050.0$ \\
& & & $4055.0-4070.0$ \\
$\Delta(4000 \AA)$ & $4000 \AA$ break & & $3750.0-3950.0$ \\
& & & $4050.0-4250.0$ \\
\hline
\end{tabular}

the line-strength indices as a function of the stellar surface temperatures.

Almost all these indices are good metallicity indicators, while they are only slightly sensitive to stellar age variations. The only relevant exception is the $\mathrm{H} \beta$ index, that is the most widely adopted optical age indicator. Like all the Balmer absorptions, the $\mathrm{H} \beta$ line appears very weak in the cold stellar types (M, K), while its intensity grows with temperature, reaching its maximum value in the spectra of A type stars. In Paper III, we will show some evidences that suggest that the $\mathrm{H} \beta$ index is not very sensitive to the ages of stellar populations younger than 1 Gyr.

We indicate as blue three indices, not present in the Lick set, in the wavelength range $3750<\lambda<$ $4200 \AA$, namely, $\mathrm{H}+\mathrm{K}(\mathrm{CaII})$ and $\mathrm{H} \delta / \mathrm{FeI}$ indices, defined by Rose $(1984,1985)$, and the $\Delta(4000 \AA)$ index defined by Hamilton (1985). The "blue" part of a galaxy spectrum is much more sensitive to the stellar population age than the "red" one (see Paper IV).

The $\mathrm{H}+\mathrm{K}(\mathrm{CaII})$ index represents the ratio between the central intensity of the $\mathrm{H}(\mathrm{CaII})+\mathrm{H} \varepsilon$ line (a blend of the $\mathrm{H}(\mathrm{CaII}) 3968.5 \AA$ with the Balmer $\mathrm{H} \varepsilon$ ) and that of $\mathrm{K}(\mathrm{CaII}) 3933.7 \AA$ line. In the same way, the definition of $\mathrm{H} \delta / \mathrm{FeI}$ index is the ratio of the central intensity of the Balmer $\mathrm{H} \delta$ line with the average value obtained from the central intensities of two FeI lines, Fe4045 and Fe4063. The spectral windows adopted to identify the centre of each of these lines are reported in Table 7. These two indices are Balmer lines measures, and they are then good age indicators (just like $\mathrm{H} \beta$ ); $\mathrm{H} \delta / \mathrm{FeI}$ is sensitive also to the metallicity parameter for its dependence from the FeI lines.

The $\Delta(4000 \AA)$ index maps the $4000 \AA$ break. It is defined as the ratio of the average fluxes (for frequency unit) measured in the spectral ranges [4050 $\AA-4250 \AA]$ and $[3750 \AA-3950 \AA]$ :

$\Delta(4000 \AA)=\frac{F_{\nu}[4050 \AA-4250 \AA]}{F_{\nu}[3750 \AA-3950 \AA]}$.

The definition of this index needs a measure of fluxes per frequency units $\left[\mathrm{Hz}^{-1}\right]$, while data are calibrated in counts per wavelength units $\left[\AA^{-1}\right]$. So, we need to multiply the
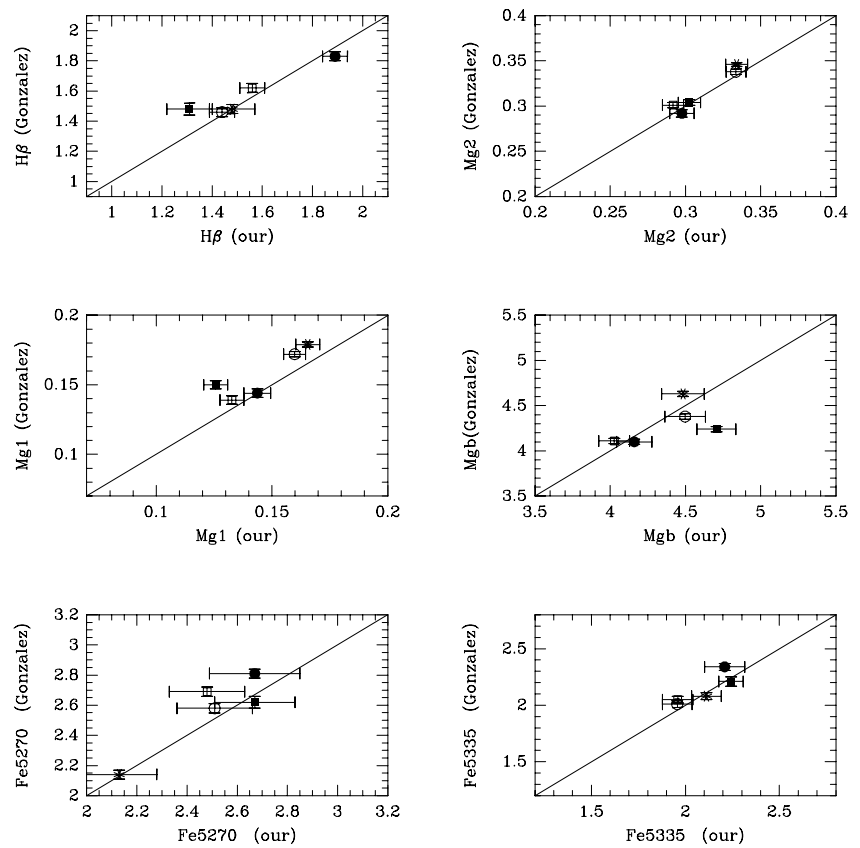

Fig. 4. Comparison between our measures (indicated as "(our)") and those of G93 (indicated as "(Gonzalez)") on a common sample of 5 template galaxies (Legenda: asterisk= NGC 7626, open square $=$ NGC 7562 , full square $=$ NGC 7785 , open circle $=$ NGC 7619 and full circle=NGC 584). In both systems, data are not corrected for velocity dispersions

ratio between fluxes $/ \AA$ by the correction factor:

$\left(\lambda_{1} / \lambda_{2}\right)^{2}=(4150 / 3850)^{2}=1.162$

where $\lambda_{1}$ and $\lambda_{2}$ represent the central wavelength of the two spectral bandpasses adopted for the $\Delta(4000 \AA)$ measure. Note that this index gives information about the stellar parameters of the turn off stars (and consequently about the mean age of the stellar population) (W92).

Spectral bandpasses of the indices have been corrected for the galaxy redshift $z$ :

$\lambda_{i}(\operatorname{corr})=\lambda_{i}(z+1)$

$\Delta \lambda(\operatorname{corr})=\Delta \lambda(z+1)$

where $\lambda_{i}$ stands for a generic bound of the spectral windows and $\lambda_{i}$ (corr) for its corresponding corrected value, $\Delta \lambda$ and $\Delta \lambda$ (corr) indicate respectively the original and the corrected spectral width. The redshift value, $z=v / c$, is directly estimated from the spectra lines.

\subsection{The conversion to the Lick-IDS system}

The Lick Group (Burstein et al. 1984; Faber et al. 1985; Burstein et al. 1986; Gorgas et al. 1993; W92; Worthey et al. 1994) has built fitting functions for 21 indices, based on a stellar spectral library of more than 400 stars. Their observations have been performed at the $3 \mathrm{~m}$ Shane Telescope (Lick Observatory) equipped with an Image 
Table 8. Stars from Lick Library

\begin{tabular}{cccccccc}
\hline HR & HD & $V$ & $B-V$ & $U-B$ & $R-I$ & Sp. class. & RV \\
\hline 1907 & 37160 & 4.09 & 0.95 & 0.64 & 0.55 & K0IIIb & 99 \\
2233 & 43318 & 5.65 & 0.50 & 0.00 & & F6V & -37 \\
2429 & 47205 & 3.95 & 1.06 & 1.01 & 0.51 & K1III & 3 \\
2503 & 49161 & 4.77 & 1.40 & 1.65 & 0.70 & K4III & 47 \\
2506 & 49293 & 4.47 & 1.11 & 1.04 & 0.55 & K0III & 11 \\
2574 & 50778 & 4.07 & 1.43 & 1.70 & 0.78 & K4III & 97 \\
2701 & 54810 & 4.92 & 1.03 & 0.78 & 0.52 & K0III & 79 \\
2854 & 58972 & 4.32 & 1.43 & 1.54 & 0.79 & K3III & 47 \\
2970 & 61935 & 3.93 & 1.02 & 0.88 & 0.52 & K0III & 11 \\
3145 & 66141 & 4.39 & 1.25 & 1.28 & 0.67 & K2III & 71 \\
3249 & 69267 & 3.52 & 1.48 & 1.77 & 0.78 & K4III & 22 \\
3418 & 73471 & 4.44 & 1.21 & 1.28 & 0.56 & K2III & 25 \\
3845 & 83618 & 3.91 & 1.32 & 1.46 & 0.67 & K2.5III & 23 \\
3994 & 88284 & 3.61 & 1.01 & 0.92 & 0.48 & K0III & 19 \\
4287 & 95272 & 4.08 & 1.09 & 1.00 & 0.55 & K0III & 47 \\
4995 & 114946 & 5.33 & 0.87 & & & G6IV & -45 \\
& 36003 & & & & 0.61 & K5V & \\
& 64606 & & & & 0.47 & G8V & \\
\hline
\end{tabular}

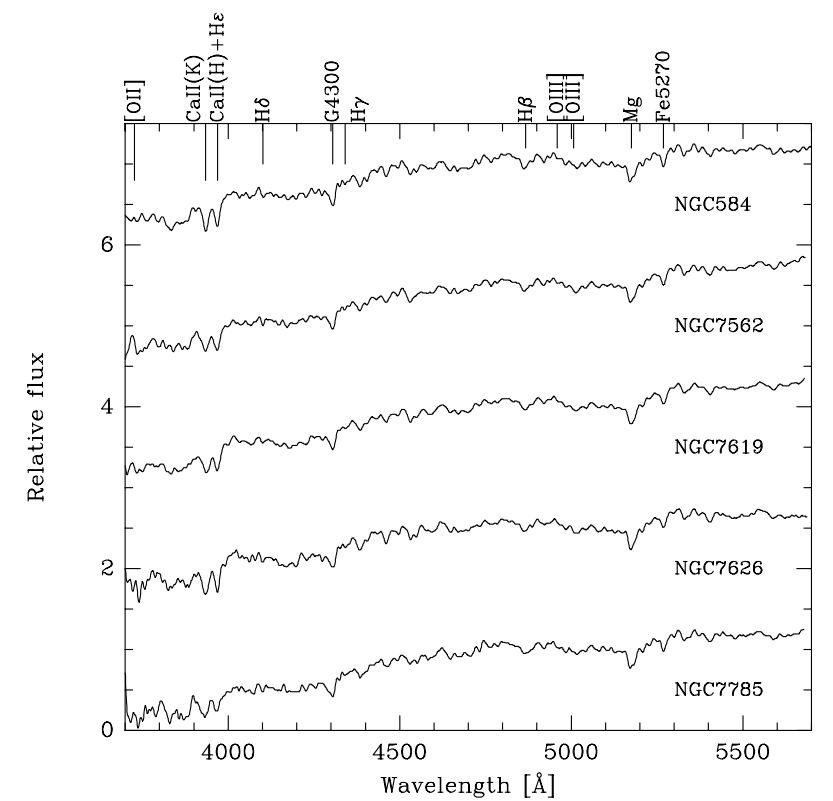

Fig. 5. Atlas of the spectra of the observed galaxies: galaxies from the G93 sample used as spectrophotometric templates. Plots are in the rest frame, and the fluxes are in arbitrary units. The most remarkable features are labelled on top

Dissector Scanner, characterized by a resolution of $8.2 \AA$ (FWHM). The present work is based on the measure of 16 red indices common to those studied by the Lick Group, for which we will use their standard fitting functions (Longhetti et al. 1997b, Paper III). In this context we need to transform our data into the "standard" LickIDS system. For this purpose, we have observed a sample of 19 stars of different spectral types (Table 8), common to the Lick library. On their spectra, once degraded to the IDS resolution, we measured the 16 red indices. The comparison between our results with those of the Lick group (Faber et al. 1985; Gorgas et al. 1993; W92) shows an acceptable agreement between the two measurement systems for some indices ( $\mathrm{H} \beta, \mathrm{Mgb}, \mathrm{Fe} 5335$, Ca4227, G4300, Fe5270), while systematic differences are observed for the others. Table 9 reports the average values of the differences found on the stellar sample between the two measurement systems.

G93 has already pointed out that residual differences are connected to the fact that while calibration of CCD data are really relative flux (i.e. corrected for the CCD response function constructed from observations of standard stars), IDS data are calibrated with a tungsten lamp as a reference source. As a consequence, IDS measurements contain the lamp contribution to the corresponding spectral features. Following G93 we have applied a shift to some indices measures in order to fully transform them in the Lick-IDS system. The adopted shifts for each index are listed in the Col. 4 of Table 9. The shifts have been applied only to the indices for which the (IDS-CCD) values have a systematic trend. G93 reports 8 "red" indices (among the 21 of the Lick set) measured on a sample of 35 stars common to the Lick library. The average differences reported by G93 are compatible with ours within errors. For comparison, in Table 9 we report also the shifts adopted by G93. A remarkable exception is represented by Fe5406, which shows a systematic scatter between our and Lick data, unlike G93 data. Figures 3a,b show the comparison 
between our fully transformed data of the sample of 19 stars and the Lick-IDS data.

\subsection{Correction of line-strength indices for velocity dispersion}

The observed spectrum of a galaxy can be regarded as a stellar spectrum (reflecting the global spectral characteristics of the galaxy) convolved with the radial velocities distribution of its stellar population. Therefore the spectral features in a galaxy spectrum are not the simple sum of the corresponding stellar features, because of the motions of the stars composing the galaxy. If we want to explain the measure of the indices in terms of the stellar composition of the galaxies, we need to correct them for the effects of the velocity dispersion. We have estimated the correction studying the behaviour of all the indices on a sample of about 80 stars. Stellar spectra (after they have been degraded to the Lick-IDS resolution) have been convolved with gaussian curves of various width in order to simulate different galactic velocity dispersions. We have considered velocity dispersions in the range $50 \mathrm{~km} \mathrm{~s}^{-1}<\sigma<300 \mathrm{~km} \mathrm{~s}^{-1}$. The results of these convolutions are reported in Table 10. Corrections for the velocity field were finally applied to the indices for which the correction itself shows a monotonic variation as a function of the velocity dispersion. This was the case for $\mathrm{H}+\mathrm{K}(\mathrm{CaII}), \mathrm{H} \beta, \mathrm{Mg} 2, \mathrm{Mgb}, \mathrm{G} 4300, \mathrm{Fe} 5270, \mathrm{Ca} 4227, \mathrm{CN}$,

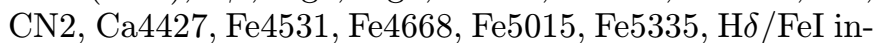
dices. The $\Delta(4000 \AA)$ behaviour is not reported in Table 10 because this index is insensitive to the spectral broadening caused by velocity dispersion, since it refers to quite large bandpasses. Mg1, Fe4383 and Fe5406 indices have not been corrected since they show a fluctuating behaviour as a function of $\sigma$ and any tentative correction can introduce a further error.

Corrections are calculated as a linear interpolation of the data in Table 10 corresponding to the actual velocity dispersion of each galaxy. Since the spectral indices refer to the nuclear region the correction is derived assuming a value of the velocity dispersion in the same region. Kinematical data adopted for the whole sample are reported in Paper II.

\subsection{Error estimation in line-strength indices}

Formally, the definition of an index is a ratio between fluxes integrated over particular bandpasses. The estimate of the error is then the propagation of the relative uncertainties in the fluxes used to calculate its value. If uncertainties of relative fluxes in different bandpasses are independent, the error is:

$$
\sigma(I)=\sqrt{\left(\frac{\partial I}{\partial f_{1}}\right)^{2} \sigma_{f 1}^{2}+\left(\frac{\partial I}{\partial f_{2}}\right)^{2} \sigma_{f 2}^{2}+\left(\frac{\partial I}{\partial f_{3}}\right)^{2} \sigma_{f 3}^{2}}
$$

where $I=I\left(f_{1}, f_{2}, f_{3}\right)$ indicates the dependence of the index on the $f_{i}$ flux measured in the three bandpasses. The derivatives of the formulas (1) and (2) give the expected uncertainty for atomic $\left(\sigma\left(I_{\mathrm{a}}\right)\right)$ and for molecular indices $\left(\sigma\left(I_{\mathrm{m}}\right)\right)$ :

$$
\begin{aligned}
& \sigma^{2}\left(I_{\mathrm{a}}\right)=\left(\frac{F_{\mathrm{I} 1, \mathrm{I} 2}}{C_{\mathrm{I}}}\right)^{2}\left[\left(\frac{\sigma\left(F_{\mathrm{I} 1, \mathrm{I} 2}\right)}{F_{\mathrm{I} 1, \mathrm{I} 2}}\right)^{2}+\right. \\
& \left.+\left(\frac{\sigma\left(F_{\mathrm{b} 1, \mathrm{~b} 2}\right)}{C_{\mathrm{I}}}\right)^{2}\left(\frac{\lambda_{\mathrm{r}}-\lambda_{\mathrm{I}}}{\lambda_{\mathrm{r}}-\lambda_{\mathrm{b}}}\right)^{2}+\left(\frac{\sigma\left(F_{\mathrm{r} 1, \mathrm{r} 2}\right)}{C_{\mathrm{I}}}\right)^{2}\left(\frac{\lambda_{\mathrm{b}}-\lambda_{\mathrm{I}}}{\lambda_{\mathrm{r}}-\lambda_{\mathrm{b}}}\right)^{2}\right]_{(5)} \\
& \sigma\left(I_{\mathrm{m}}\right)=\frac{2.5 \times 10^{0.4 I_{\mathrm{m}}}}{2.3026\left(\lambda_{\mathrm{I} 2}-\lambda_{\mathrm{I} 1}\right)} \sigma\left(I_{\mathrm{a}}\right) \\
& \lambda_{\mathrm{I}}=\frac{\left(\lambda_{\mathrm{I} 1}+\lambda_{\mathrm{I} 2}\right)}{2} \\
& \lambda_{\mathrm{r}}=\frac{\left(\lambda_{\mathrm{r} 1}+\lambda_{\mathrm{r} 2}\right)}{2} \\
& \lambda_{\mathrm{b}}=\frac{\left(\lambda_{\mathrm{b} 1}+\lambda_{\mathrm{b} 2}\right)}{2} \\
& C_{\mathrm{I}}=C\left(\lambda_{\mathrm{I}}\right) \\
& F_{\mathrm{I} 1, \mathrm{I} 2}=\int_{\lambda_{\mathrm{I} 1}}^{\lambda_{\mathrm{I} 2}} F(\lambda) \mathrm{d} \lambda\left(\frac{\sigma\left(F_{\mathrm{I} 1, \mathrm{I} 2}\right)}{F_{\mathrm{I} 1, \mathrm{I} 2}}\right)^{2}=\frac{1}{\int_{\lambda_{\mathrm{I} 1}}^{\lambda_{\mathrm{I}}} \frac{F^{2}(\lambda)}{\sigma^{2}(\lambda)}} \\
& \sigma^{2}\left(F_{\mathrm{b} 1, \mathrm{~b} 2}\right)=\frac{F_{\mathrm{b}}^{2}}{\int_{\lambda_{\mathrm{b} 1}}^{\lambda_{\mathrm{b} 2}} \frac{F^{2}(\lambda)}{\sigma^{2}(\lambda)} \mathrm{d} \lambda} i \\
& \sigma^{2}\left(F_{\mathrm{r} 1, \mathrm{r} 2}\right)=\frac{F_{\mathrm{r}}^{2}}{\int_{\lambda_{\mathrm{r} 1}}^{\lambda_{\mathrm{r} 2}} \frac{F^{2}(\lambda)}{\sigma^{2}(\lambda)} \mathrm{d} \lambda}
\end{aligned}
$$

where $F(\lambda)$ is the spectral flux and $\sigma^{2}(\lambda)$ the flux variance as a function of wavelength. The flux variance is calculated from the wavelength calibrated frames, transforming the ADU counts into the corresponding photons counts. In the present work, the transformation is given by:

$$
I(\text { col }, \text { row })[\text { photons }]=\operatorname{ADU}(I(\text { col }, \text { row })[\text { counts }])
$$

with $1 \mathrm{ADU}=1.4 \mathrm{e}^{-}$. Summing the Read Out Noise (RON) characterizing the $\mathrm{CCD}$, we calculate the variance frame:

$$
\sigma^{2}(\mathrm{col}, \text { row })=I(\text { col }, \text { row })[\text { photons }]+\sigma_{\text {RON }}^{2}
$$

where $\sigma_{\text {RON }}^{2}=6.5^{2}=42.25$. From this frame we extracted a $1 \mathrm{D}$ spectrum with the same parameters adopted to extract the fully calibrated spectrum of the corresponding object. This represents the $\sigma^{2}(\lambda)$ in the previous formulas. In fact, this variance describes the uncertainty that characterizes the measurements of flux for each spectrum, i.e. it represents the statistical error of the flux measurement pixel by pixel, which is present even if the photometric system is perfectly reproducible.

Figure 4 shows the comparison between our and G93 data, on a subset of 6 red indices, including the $\mathrm{H} \beta$ and 


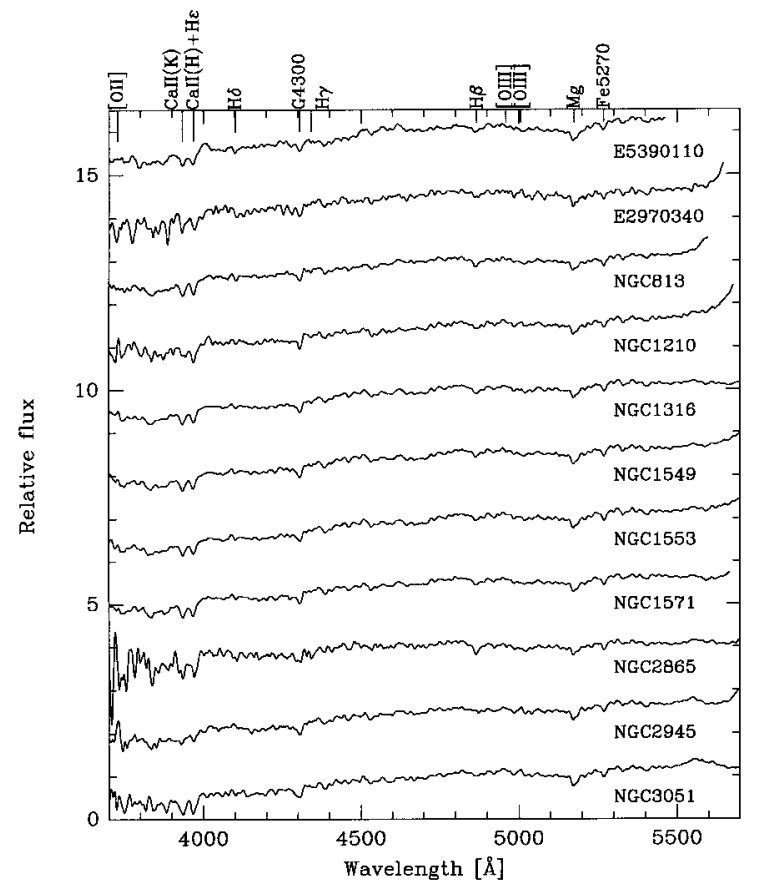

a)

Fig. 6. Same as Fig. 5 for shell galaxies

$\mathrm{Mg}$ and Fe indices which will be used to reconstruct the star formation history of the galaxies. Our line-strength indices are in agreement, within the errors, with those of G93.

Actually the measurement of a line-strength index does not need an absolute flux calibrated spectrum, since only flux ratios enter in its definition. The estimate of the error calculated adopting (6) and (7) then corresponds to an upper limit of the real uncertainty that characterizes our index measurement. In fact, it does not take into account the consequences of the gaussian filter applied to our data before measurements of the index since the data quality is enhanced by this treatment to the spectral resolution cost. A more realistic way to calculate the uncertainty that affects our indices measures could then be to refer to a quantity that represents how the corresponding spectral feature is visible within a noisy continuum.

We substitute Eqs. (1) and (2) with their approximations obtained using constant average values of the fluxes in the integrals:

$I_{\mathrm{a}}=\left(\lambda_{2}-\lambda_{1}\right)\left(1-\frac{f_{\mathrm{I}}}{f_{\mathrm{C}}}\right)$

$I_{\mathrm{m}}=-2.5 \log _{10}\left(\frac{f_{\mathrm{I}}}{f_{\mathrm{C}}}\right)$

where $f_{\mathrm{I}}$ is the flux average value in the central bandpass (indicated by $\lambda_{1}$ and $\lambda_{2}$ ), and $f_{\mathrm{C}}$ is the average continuum flux calculated with (3) at $\lambda_{\mathrm{I}}=\left(\lambda_{1}+\lambda_{2}\right) / 2$.

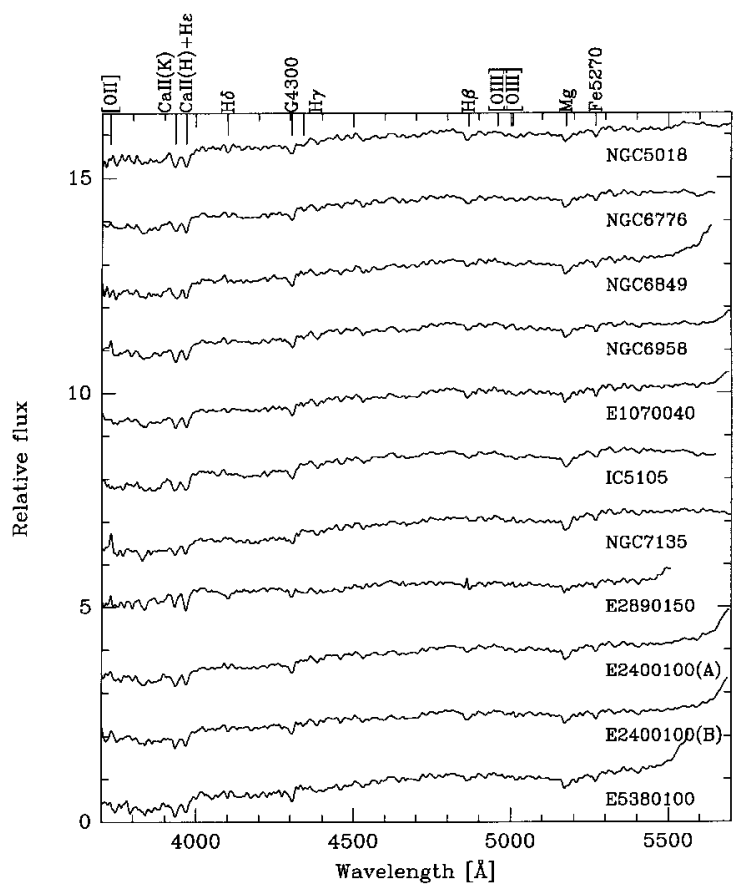

b)
In this way, Eqs. (6) and (7) become:

$$
\begin{gathered}
\sigma^{2}\left(I_{\mathrm{a}}\right)=\left(\lambda_{2}-\lambda_{1}\right)^{2}\left(\frac{f_{\mathrm{I}}}{f_{\mathrm{C}}}\right)^{2}\left[\left(\frac{\sigma\left(f_{\mathrm{I}}\right)}{f_{\mathrm{I}}}\right)^{2}+\right. \\
\left.+\left(\frac{\sigma\left(f_{\mathrm{r}}\right)}{f_{\mathrm{C}}}\right)^{2}\left(\frac{\lambda_{\mathrm{b}}-\lambda_{\mathrm{I}}}{\lambda_{\mathrm{r}}-\lambda_{\mathrm{b}}}\right)+\left(\frac{\sigma\left(f_{\mathrm{b}}\right)}{f_{\mathrm{C}}}\right)^{2}\left(\frac{\lambda_{\mathrm{r}}-\lambda_{\mathrm{I}}}{\lambda_{\mathrm{r}}-\lambda_{\mathrm{b}}}\right)\right] \quad(9) \\
\sigma^{2}\left(I_{\mathrm{m}}\right)=(1.0875)^{2}\left[\left(\frac{\sigma\left(f_{\mathrm{I}}\right)}{f_{\mathrm{I}}}\right)^{2}+\left(\frac{\sigma\left(f_{\mathrm{r}}\right)}{f_{\mathrm{C}}}\right)^{2}\left(\frac{\lambda_{\mathrm{b}}-\lambda_{\mathrm{I}}}{\lambda_{\mathrm{r}}-\lambda_{\mathrm{b}}}\right)+\right. \\
\left.+\left(\frac{\sigma\left(f_{\mathrm{b}}\right)}{f_{\mathrm{C}}}\right)^{2}\left(\frac{\lambda_{\mathrm{r}}-\lambda_{\mathrm{I}}}{\lambda_{\mathrm{r}}-\lambda_{\mathrm{b}}}\right)\right] .
\end{gathered}
$$

We then need to know only the average value of flux uncertainties, $\sigma\left(f_{\mathrm{I}}\right), \sigma\left(f_{\mathrm{r}}\right)$ and $\sigma\left(f_{\mathrm{b}}\right)$, substituting the variance function $\sigma^{2}(\lambda)$. These average values can be calculated as the dispersion of the flux around its average value in the corresponding bandpass. Errors computed following the procedure outlined above are reported in Tables 11, 12 and 13.

A remarkable exception is the error estimate for the $\mathrm{H}+\mathrm{K}(\mathrm{CaII})$ and $\mathrm{H} \delta / \mathrm{FeI}$ indices. Their definitions are based on the flux values corresponding to specific single pixels, and so their uncertainties are calculated propagating the measure of the errors on the flux extracted from the variance spectra (referred before to $\sigma^{2}(\lambda)$ ) at those pixels, decreased by a factor of about 2.8 (which corresponds to the square root of the number of the pixels involved in the gaussian filter windows). 

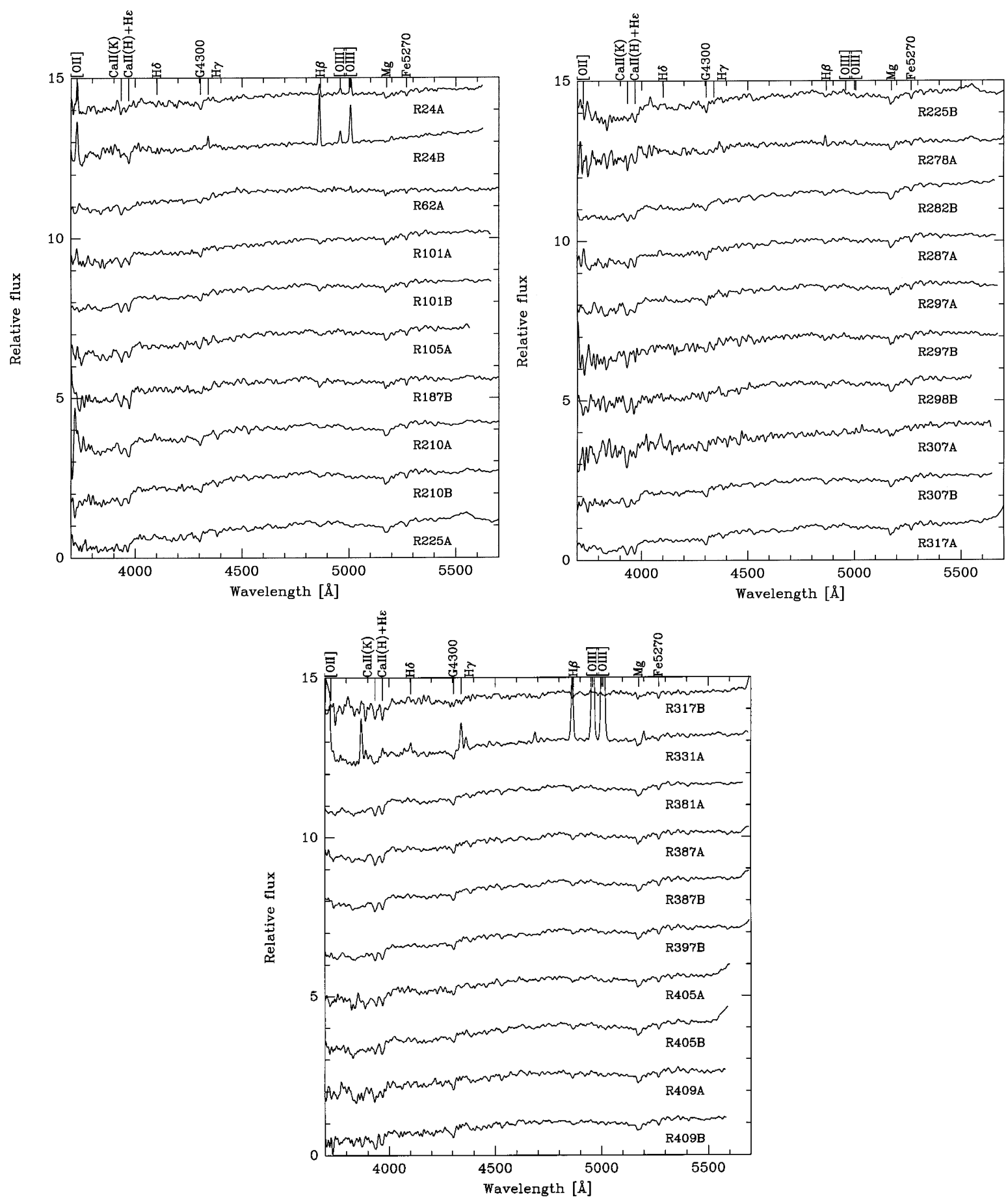

Fig. 7. Same as Fig. 5 for pair members

\subsection{Comparisons with line-strength nuclear indices for galaxies in the G93 sample}

Table 11 reports our nuclear line-strength indices on the 5 "template" galaxies belonging to the G93 sample. The Table lists also the differences between our results and those achieved by Worthey (1996, private communication), on the common subset of 16 "red" indices. Worthey IDS data are of poorer quality than our CCD ones, and than they are characterized by greater uncertainties (see also G93).

The comparison is made using line-strength indices before the correction for the velocity dispersion, since this latter has been obtained by G93 with a procedure different from that described in Sect. 4.3. Our corrections for velocity dispersion are nearly identical to those of G93 for $\mathrm{H} \beta$ and $\mathrm{Mg}$ indices, but our procedure led to corrections which are of a few percent smaller than in G93 for the Fe 
lines-strength indices. In Paper II we show that our estimate of velocity dispersions for template galaxies are in very good agreement with G93: $\left\langle\left(\sigma_{\mathrm{G} 93}-\sigma_{\text {our }}\right)>=9 \pm 9\right.$. These differences will be taken into account during the comparison of the two set of data in Paper III.

Data reported in Tables 12 and 13, relative to the indices measurements in our sample galaxies, have not been corrected for a possible contamination from the emission components. Paper II will address the issue of an analysis of these emission lines and a forthcoming paper that of a study of the absorption lines that takes into account their contamination due to filling.

\section{Summary}

We present the first of a series of papers (Longhetti et al. $1997 \mathrm{a}, \mathrm{b}, \mathrm{c})$ that will study the star formation history in early-type galaxies. In the present paper, we present a sample of 20 shell galaxies and 32 pair members in low density environments that is studied for this purpose. Most of the objects show present/past interaction signatures.

The paper describes the observations and the reduction of the spectrophotometric data obtained from spectra in the wavelength range of $3700 \AA<\lambda<5700 \AA$ at $2.1 \AA$ FWHM resolution.

A set of $16 \mathrm{red}(\lambda>4200 \AA)$ nuclear $\left(5^{\prime \prime}\right)$ line-strength indices defined by the Lick Group has been obtained and converted into the Lick-IDS "standard" system. 5 reference galaxies, selected from the G93 sample, are also included to test the results of the procedure.

Three blue $(\lambda<4200)$ nuclear line-strength indices, namely $\Delta(4000 \AA), \mathrm{H}+\mathrm{K}(\mathrm{CaII})$ and $\mathrm{H} \delta / \mathrm{FeI}$, have been also determined with the purpose of tracing recent starbursts in the galaxies history.

The kinematics of the gas and of the stellar components are the subject of the Paper II. Stellar kinematical data (organized and discussed in the Paper II) have been used to correct present line-strength indices for velocity dispersion.

A fraction of the galaxies of the present study shows detectable emission lines, as can be deduced from the Atlas of the spectra (Figs. 5 to 7 ). A discussion about the physical conditions of the (e.g. LINER properties of the nucleus, ect.) is also given in the Paper II. Line-strength indices suffer the contamination caused by emission lines. G93 corrected the $\mathrm{H} \beta$ absorption line-strength for the filling due to the $\mathrm{H} \beta$ emission by means of a relation between the $\mathrm{H} \beta$ line emission strength and that of the [OIII] line at $5007 \AA$. Namely $\Delta \mathrm{H} \beta=0.7[\mathrm{OIII}]$. The validity of this correction has been questioned by Carrasco et al. (1995). We discuss the correction of the line-strength indices for the emission line filling in a forthcoming papera forthcoming paper.
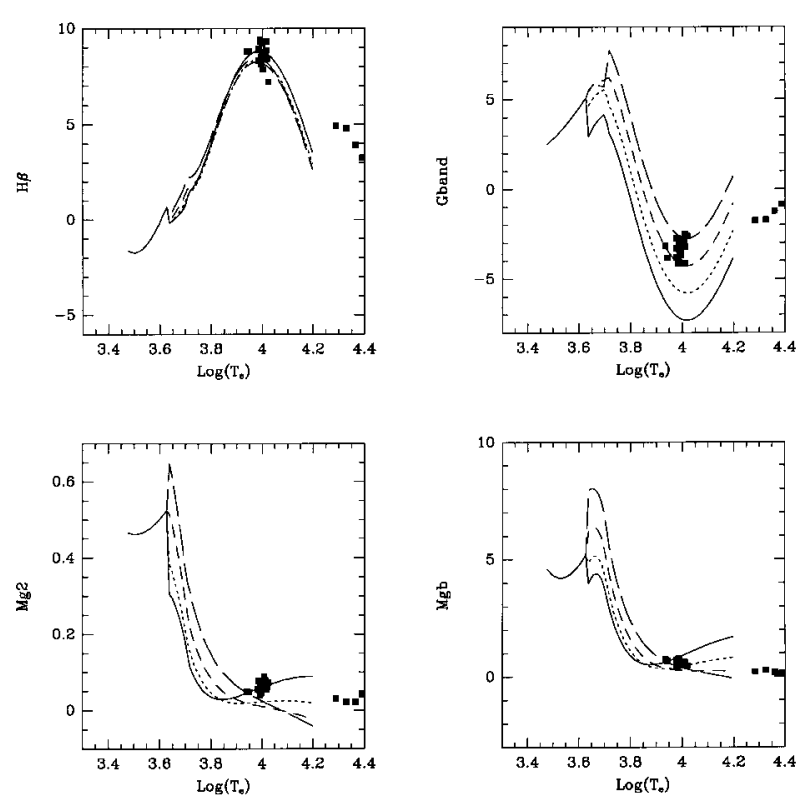

Fig. 8. Fitting functions of W92 for gravity $g=4.5$ and different metallicities (lines: solid line $[\mathrm{Fe} / \mathrm{H}]=-2$, short dash $[\mathrm{Fe} / \mathrm{H}]=-1$, medium dash $[\mathrm{Fe} / \mathrm{H}]=0$, long dash $[\mathrm{Fe} / \mathrm{H}]=1)$. Full squares represent our measures on a sample of 17 hot stars, listed in Table 14. Note that in the case of the $\mathrm{H} \beta$ index, fitting functions from W92 do not match our observations

\section{Appendix A}

Line-strength indices of a sample of 17 hot stars $(T>$ $10000 \mathrm{~K})$. Toward an extension of the Lick Fitting Functions

The aim of the present work (cf. also Papers II and III) is that of looking for the presence of recent star formation events connected to interaction, that can have involved only a few percentages of the total mass of the galaxies. Both observations and numerical/hydrodynamical simulations (see for references Barnes \& Hernquist 1992) indicate that induced star formation is a possible effect of the interaction.

Young stellar populations, if present, are made recognizable in a galaxy by the presence of very hot stars $(T>10000 \mathrm{~K})$. These kinds of stars are under-represented in the sample from which W92 derived his fitting functions. Consequently, the knowledge of the indices behaviour in the range of stellar temperatures greater than $10000 \mathrm{~K}$ is a necessary step in the construction of suitable fitting functions.

We have observed 17 hot stars (whose parameters are listed in Table 14) in order to test the Lick standard fitting functions in the high temperature regime. Figure 8 shows a comparison between Lick fitting functions for some indices and stellar data collected in the present work. In the case of the $\mathrm{H} \beta$ index, the only classic age indicator in the "red" part of the spectrum, it is quite evident that the standard fitting functions do not match our observations. This suggests that the extrapolation starting from lower 
values of stellar temperatures towards higher ones need to be better investigated on a larger sample. We have used our new data to construct a tentative extension of the Lick Group fitting functions for the 16 common "red" indices. Table 13 lists the coefficients of these new extensions, obtained through a linear interpolation of the values starting from $\log T_{\mathrm{e}}=4$ for the different metallicities and gravities.

Acknowledgements. ML acknowledges the kind hospitality of the Brera Observatory and of the Padova Observatory during her PhD Thesis. We thank Ginevra Trinchieri for the careful reading of the manuscript. ML, AB and $\mathrm{CC}$ acknowledge the support by the European Community under TMR grant ERBFMRX-CT96-0086.

\section{References}

Barnes J., 1997, in SAAS-FEE

Barnes J.E., Hernquist L.E., 1992, ARA\&A 30, 705

Bender R., 1997, in The Second Stromlo Symposium: The Nature of Elliptical Galaxies, Arnaboldi M. et al. (eds.), p. 11

Bender R., Saglia R.P., Ziegler B., 1996, The early Universe with the VLT, Bergeron J. et al. (eds.). Springer Verlag, Berlin, (in press)

Bica E., Alloin D., 1987, A\&A 186, 49

Bower R.G., Ellis R.S., Rose J.A., Sharples R.M., 1990, AJ 99, 530

Bressan A., Chiosi C., Tantalo R., 1996, A\&A 311, 425

Burstein D., Heiles C., 1982, AJ 87, 1165

Burstein D., Faber S.M., Gaskell C., Krumm N., 1984, ApJ 287, 586

Burstein D., Faber S.M., Gonzalez J.J., 1986, AJ 91, 1130

Buzzoni A., 1989, ApJS 71, 817

Buzzoni A., 1995, ApJS 98, 69

Buzzoni A., Gariboldi G., Mantegazza L., 1992, AJ 103, 1814

Carrasco L., Buzzoni A., Salsa M., Recillas-Cruz E., 1995, Fresh Views on Elliptical Galaxies, Buzzoni A., Renzini A., Serrano A. (eds.) ASP Conf. Ser. 86, 129

Carter D., Prieur J.L., Wilkinson A., Sparks W.B., Malin D.F., 1988, MNRAS 235, 813

Charlot S., Silk J., 1994, ApJ 432, 453

Davies R.L., 1995, in Fresh Views on Elliptical Galaxies, Buzzoni A., Renzini A., Serrano A. (eds.) ASP Conf. Ser. 86,175

de Carvalho R.R., Djorgovski S.G., 1992, ApJ 389, L49

Djorgovski S.G., Phare M.A., de Carvalho R.R., 1995, Fresh Views on Elliptical Galaxies, Buzzoni A., Renzini A., Serrano A. (eds.) ASP Conf. Ser. 86, 129

Dupraz C., Combes F., 1986, A\&A 166, 53

Faber S.M., Friel E.D., Burstein D., Gaskell C.M., 1985, ApJS 57,711
Gonzalez J.J., 1993, Ph.D. Thesis, Univ. of California, Santa Cruz: G93

Gorgas J., Faber S.M., Burstein D., Gonzalez J.J., Courteau S., Prosser C., 1993, ApJS 86, 153

Griffith M.R., Wright A.E., Burke, B.F., Ekers R.D., 1994, ApJS 91, 111

Governato F., Gardner J., Stadel J., Quinn T., Lake G., 1997, ApJ (submitted)

Jorgensen I., Franx M., 1995, Fresh Views on Elliptical Galaxies, Buzzoni A., Renzini A., Serrano A. (eds.) ASP Conf. Ser. 86, 139

Hamilton D., 1985, ApJ 297, 371

Hernquist L., Quinn P.J., 1989, ApJ 342, 1

Lauberts A., Valentijn E.A., 1989, The Surface Photometry Catalogue of the ESO-Uppsala Galaxies, ESO: ESO-LV

Leonardi A.J., Rose J.A., 1996, AJ 111, 182

Longhetti M., Rampazzo R., Bressan A., Chiosi C., 1997a, A\&AS (in press) (Paper II)

Longhetti M., Bressan A., Chiosi C., Rampazzo R., 1997b, A\&AS (in preparation) (Paper III)

Longhetti M., Bressan A., Chiosi C., Rampazzo R., 1998 , A\&AS (in preparation) (Paper IV)

Malin, Carter D., 1983, ApJ 274, 534: MC83

Moore B., Katz N., Lake G., Dressler A., Oemler A., 1996, Nat 379,613

Pickles A.J., 1985, ApJ 296, 340

Quinn P.J., 1984, ApJ 279, 596

Reduzzi L., Rampazzo R., 1995, Astr. Lett. Comm. 30, 1: RR95

Reduzzi L., Longhetti M., Rampazzo R., 1996, MNRAS 282, 149

Rose J.A., 1984, AJ 89, 1238

Rose J.A., 1985, AJ 90, 1927

Rose J.A., 1995, Fresh Views on Elliptical Galaxies, Buzzoni A., Renzini A., Serrano A. (eds.) ASP Conf. Ser. 86, 157

Rose J.A., Bower R.G., Caldwell N., Ellis R.S., Sharples R.M., Teague P., 1994, AJ 108, 2054

Schweizer F., 1992, Structure, dynamics and chemical evolution of elliptical galaxies, Danziger I.J., Zeilinger W.W., Kjar K. (eds.) p. 651

Thomson R.C., Wright, 1990, MNRAS 217, 122

Thomson R.C., 1991, MNRAS 253, 256

Tully R.B., 1988, Nearby Galaxy Catalogue. Cambridge University Press

Toomre A., Toomre J., 1972, ApJ 178, 623

Toomre A., 1977, in The Evolution of Galaxies and Stellar Populations, Tinsley B.M, Larson R.B. (eds.). New Haven: Yale University Press, p. 401

Worthey G., 1992, Ph.D. Thesis, Univ. California, Santa Cruz: W92

Worthey G., 1994, ApJS 95, 107

Worthey G., Faber S.M., Gonzalez J.J., Burstein D., 1994, ApJS 94, 687 